\title{
Simultaneous encoding of direction at a local and global scale
}

\author{
SCOTT N. J. WATAMANIUK \\ Wright State University, Dayton, Ohio \\ and \\ SUZANNE P. MCKEE \\ Smith-Kettlewell Eye Research Institute, San Francisco, Califormia
}

\begin{abstract}
Human observers can simultaneously encode direction information at two different scales, one local (an individual dot) and one global (the coherent motion of a field of dots distributed over a $10^{\circ}$ diameter display). We assessed whether encoding global motion would preclude the encoding of a local trajectory component and vice versa. In the present experiments, a large number (100-150) of dots were randomly assigned directions in each frame from a uniform distribution of directions spanning a range of $160^{\circ}$ to create global motion in a single direction (Williams \& Sekuler, 1984). Amidst these background dots, 1 dot moved in a consistent direction (trajectory) for the duration of the display. The direction of this "trajectory dot" was similar to the mean direction of the distribution of directions determining the movement of the background dots. Direction discrimination for both the global motion and the trajectory was measured, using the method of constant stimuli, under precued and postcued partial report conditions. A low- or high-frequency $85-\mathrm{msec}$ tone signaled which motion the subject was to judge. In the precue condition, the tone was presented 200 msec before the onset of the stimulus, whereas in the postcue condition, the tone was presented immediately after the offset of the stimulus. Direction discrimination thresholds for both global and local motion in the postcued condition were not significantly different from those obtained in the precued condition. These results suggest that direction information for both global and local motion is encoded simultaneously and that the observer has access to either motion signal after the presentation of a stimulus.
\end{abstract}

As we navigate through our environment, our visual system is bombarded with motion information at many different scales. At the largest scale - the entire visual fielda stationary background will produce an optic flow field (Gibson, 1966) that provides information about the layout of the surroundings. At a smaller scale, an individual moving object will trace out a smooth path that is superimposed upon the background motion. The smoothness of the object's path is due to inertia, which restricts a moving object from rapidly changing its direction or speed. How does our visual system deal with information about the background's motion and about the motion of an individual object on that background? Can we identify the motion of the moving object while simultaneously processing the backgound's motion?

The present experiments were designed to determine how well the human visual system encodes simultaneously presented background motion and individual element motion by measuring the precision of direction discrimination judgments. In the past, many studies evaluating humans' abilities to process information at different scales

This research was supported by AFOSR Grant F49620-95-1-0265. Correspondence should be addressed to S. N. J. Watamaniuk, Psychology Department, Wright State University, Dayton, OH 45435 (e-mail: swatamaniuk@wright.edu). were couched in terms of global precedence, the finding that global features are extracted earlier or faster than more local features (Navon, 1977).

Since Navon's (1977) original paper, many researchers have examined the global precedence phenomenon, often using a stimulus of small shapes or letters-the local information - arranged to form a large shape or letter-the global information (e.g., Hughes, Layton, Baird, \& Lester, 1984; LaGasse, 1993; Martin, 1979; Miller, 1981; Navon, 1981, 1991; Paquet \& Merikle, 1984; Pomerantz, 1983; Robertson, Egly, Lamb, \& Kerth, 1993). In these studies, global precedence has been defined in two different ways. Some researchers have used the term to refer to the interference that a global form has on an observer's ability to respond to a component local form (e.g., Hoffman, 1980; Miller, 1981; Pomerantz, 1983). Others, such as Navon (1981) and Ward (1982), have a more general definition of global precedence, using the term to refer to "the theoretical hypothesis that global features have temporal precedence during microgenesis of the percept; that is, somehow they are extracted from the visual array earlier, or more rapidly, than are more local aspects" (Ward, 1983, p. 542). Despite these two slightly differing definitions, it is generally accepted that "global" and "local" are relative: Global features do not have to encompass the whole visual field or a certain area of visual space (Navon \& Norman, 1983). In addition, both definitions suggest that the 
effect of global precedence should be observed in the time it takes for observers to respond to global and local features. That is, whether global features interfere with responses to or are extracted more rapidly than local features, response times to the local features should be longer than those to global features. Most previous studies of global precedence have used reaction time as the dependent measure, in conditions expected to provide a temporal advantage for responding to either the global or the local features.

In this paper, we take a different approach to the global precedence question. Rather than measuring the time it takes to respond to a given feature in our display, we controlled stimulus duration and measured the precision with which observers could make judgments about a given feature. Thus, we were not investigating processing time or the temporal order of processing of global and local features, but rather the integrity of the global and local information obtained within a specified duration.

Although we did not investigate the typical global precedence phenomenon, we will use the terms global and local in much the same way that Navon (1977) and others have used them. Specifically, we use the term global flow (or global motion) to refer to the coherent motion percept produced by a background of up to 150 dots that were assigned randomly their direction of motion from frame to frame from a distribution of directions spanning $160^{\circ}$. This global motion results from the integration of the motion information of each component dot over space and thus is analogous to the global forms used in global precedence studies (Watamaniuk, Sekuler, \& Williams, 1989; Williams \& Sekuler, 1984). The term local motion refers to the motion of an individual dot in the motion display and thus is analogous to the local forms used in global precedence studies.

\section{EXPERIMENT 1 Effects of Cuing}

In the first experiment, direction discrimination thresholds were evaluated for global flow and for an embedded single dot moving on a fixed trajectory with a stimulus duration limited to $167 \mathrm{msec}$. Thresholds were obtained under three different temporal cuing conditions: (1) Precueconstant: Before a block of trials, observers were told to judge the direction of either the global flow or the trajectory for every trial in the block. (2) Precue-random: Within a single block, the motion to be judged was chosen randomly each trial. An 85-msec tone was presented $200 \mathrm{msec}$ before the onset of the stimulus to indicate which motion the observer was to judge in that trial. A low-frequency tone indicated that the observer was to judge the direction of global flow, and a high-frequency tone indicated that the observer was to judge the direction of the trajectory. (3) Postcuerandom: Within a single block, the motion to be judged was chosen randomly for each trial. Immediately after the offset of the stimulus, an 85 -msec tone was presented to indicate which motion - global flow or trajectory-the observer was to judge in that trial. This procedure is modeled after Sperling's (1960) partial report method.

\section{Method}

Observers. Three observers participated in this experiment-the two authors and another experienced psychophysical observer who was naive to the hypothesis being tested. Observers ranged in age from 30 to 52 years and all had normal or corrected-to-normal visual acuity.

Stimuli. Stimuli were dynamic random-dot cinematograms in which each dot moved with a constant step size $\left(0.27^{\circ}\right)$ from frame to frame. Stimuli contained a variable number of dots that took independent 2-D random walks. In every frame, each dot's displacement was chosen randomly from a uniform distribution of directions, spanning $160^{\circ}$, and was independent of both its previous displacements and the displacements of other dots. When a field of random dots moves in this fashion, observers perceive the whole field of dots moving en masse in the direction of the mean of the distribution (Watamaniuk et al., 1989; Williams \& Sekuler, 1984). The mean direction of the distribution was one of five directions $\left(80^{\circ}, 85^{\circ}, 90^{\circ}, 95^{\circ}\right.$, or $100^{\circ}$ ), chosen randomly for each trial. Embedded within this field of dots taking 2-D random walks was 1 dot that moved on a fixed trajectory; that is, it moved in a constant direction over time. The trajectory dot moved with the same step size as the other dots, in one of 10 directions $\left(86^{\circ}, 88^{\circ}, 90^{\circ}, 92^{\circ}, 94^{\circ}, 266^{\circ}, 268^{\circ}, 270^{\circ}, 272^{\circ}\right.$, or $\left.274^{\circ}\right)$, chosen randomly for each trial. Using trajectories that went either upward or downward with the same angular difference from vertical made the orientation of the path traced out by the trajectory an unreliable cue for the discrimination task. The trajectory dot was positioned so that its motion path was centered vertically with the fixation spot in the middle of the display. However, to ensure that the fixation spot did not provide any orientation cue for the trajectory, the trajectory was randomly positioned horizontally to fall within $\pm 0.36^{\circ}$ of fixation. Figure 1 shows a scale schematic representation of the stimuli.

Stimuli were displayed, under computer control via A/D converters, on an $x-y$ cathode ray tube display (CRT) with a $10^{\circ} \times 10^{\circ}$ square plotting area equipped with a 44 phosphor. Observers viewed the CRT through a $10^{\circ}$-diameter circular mask that reduced the visible area from $100 \mathrm{deg}^{2}$ to $78.5 \mathrm{deg}^{2}$. Since dots were plotted uniformly over the $100-\mathrm{deg}^{2}$ area, the mask allowed only $78.5 \%$ of the dots to be seen at any one time. Viewing distance was $57 \mathrm{~cm}$ and a fixation spot located at the center of the screen was continuously visible. The height of the CRT was set so that the center of the aperture was approximately at eye level. Stimuli were presented at a frame rate of $60 \mathrm{~Hz}$ and each stimulus dot subtended $0.07^{\circ}$. All experiments took place with the overhead room lights on, creating a background luminance of $33 \mathrm{~cd} / \mathrm{m}^{2}$. Dot luminance was $72 \mathrm{~cd} / \mathrm{m}^{2}$. This value was obtained by plotting a matrix of nonoverlapping dots (centerto-center spacing was $0.08^{\circ}$ ) at the same frame rate as that used in the present experiments. The luminance of this matrix was then measured with a Minolta luminance meter. Because of the decay rate of the phosphor and the high background luminance, the luminance of each dot decreased to the background luminance within $10 \mathrm{msec}$. Pushbuttons connected to the computer initiated each trial and signaled observer responses.

Procedure. The number of dots creating global flow was determined separately for each observer so that the detectability of the trajectory in our usual experimental conditions (i.e., a duration of $167 \mathrm{msec}$, or 10 frames) was approximately $95 \%$. This ensured that performance differences between observers and conditions was not due to the trajectory being differentially detectable. Details of the experimental procedure used for measuring the detectability of trajectory motion can be found in Watamaniuk, McKee, and Grzywacz (1995). The number of background dots used for Observer S.M. was 100 (94\% detectability), for Observer D.T., 125 (93\% detectability), and for Observer S.W., 150 (93.5\% detectability). These numbers 

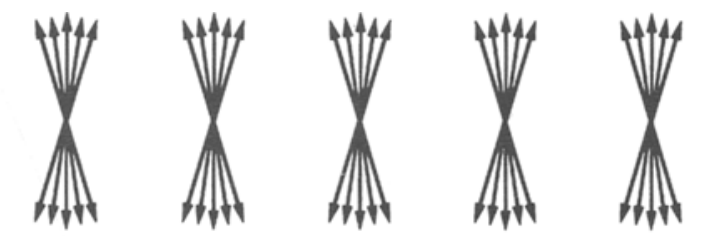

\section{Trajectory Directions}
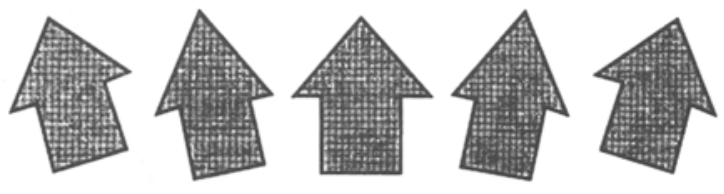

\section{Global Directions}

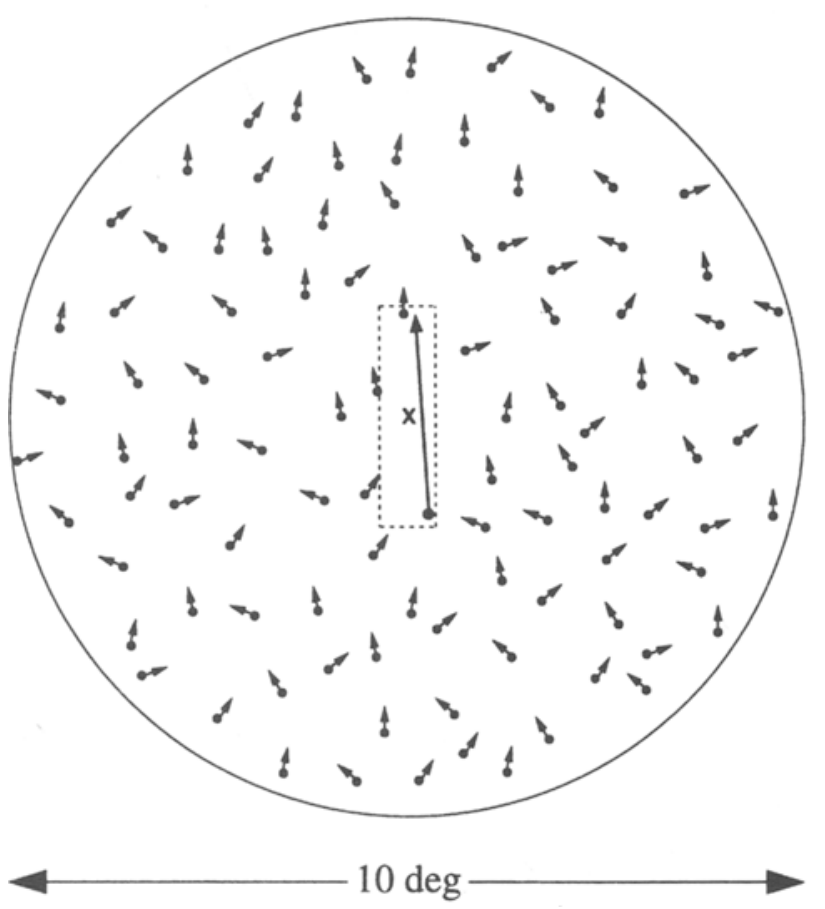

Figure 1. Schematic depiction of a random-dot stimulus with 100 background dots and 1 trajectory dot. The small and large arrows above the stimulus demonstrate that the 10 different trajectory directions were completely crossed with the five global directions. The stimuli were shown through a $10^{\circ}$-diameter aperture with a constantly visible centered fixation spot (represented by the $X$ ). The long arrow in the center of the display represents the path that a trajectory dot could have traveled in one trial (10 frames). The length of the arrows on the background dots represents the displacement taken in a single frame of motion. The dashed rectangle surrounding the trajectory represents the region of the display where the trajectory randomly appeared. Background dots were not restricted from traveling through the central region and could cross the path of the trajectory.

refer to the number of dots plotted over the entire plotting area of 100 $\operatorname{deg}^{2}$; through the $10^{\circ}$-diameter mask, only $78.5 \%$ of these dots were visible.

In the discrimination experiments, observers judged the direction of either the global motion or the trajectory dot within a two-alternative forced-choice paradigm. The method of constant stimuli was used. In each trial, one stimulus was shown and the observer judged whether the global flow or the trajectory moved to the left or right of upward. Feedback was provided. Each experimental run consisted of 200 trials, 4 for each pairing of the 10 trajectory and 5 global flow directions (see Figure 1). Each observer completed between 200 and 600 practice trials of each experimental condition prior to data collection. These practice trials were to accustom the observers to the task and to associate the tones with the motion to be judged. In ad- dition, 10 to 20 practice trials were typically run at the beginning of an experimental session to remind the observer of the stimulus and task. The percentage of trials in which the motion was judged as moving to the right of upward was recorded for each stimulus. The percent rightward judgments were fit with a cumulative normal by probit analysis (Finney, 1971). The stimulus increment-that is, the change in direction of the trajectory or global flow required to change performance from $50 \%$ to $75 \%$-was evaluated from the fitted function and used as the direction discrimination threshold. At least three runs were completed for each condition. Reported performance values were evaluated by taking the average of the thresholds evaluated for separate runs. Error bars on all graphs are \pm 1 standard errors reflecting the between-run variability for each condition and observer. 


\section{Results}

To facilitate our analysis of the pre- and postcued conditions, we performed an independent analysis of variance (ANOVA) on each of the precue-constant trajectory and global flow data sets. We examined whether the direction of the unjudged motion affected the precision of the judged motion (i.e., whether the direction of global flow affected trajectory discrimination thresholds, and vice versa). For this analysis, the data for each pairing of global and trajectory direction, for each observer, were summed across the three experimental runs. Thus, for each observer, we obtained 10 psychometric functions reflecting global direction discrimination ( 1 for each trajectory direction) and 10 psychometric functions reflecting trajectory direction discrimination ( 2 for each global direction, one measured with upward-moving trajectories and one with downwardmoving trajectories). Probit analysis was used to evaluate a single-direction discrimination threshold for each psychometric function. Figure 2 shows the trajectory and global direction discrimination data for each observer. The discrimination thresholds for the global motion are in line with previous research (Watamaniuk \& Sekuler, 1992; Watamaniuk et al., 1989). Thresholds for the trajectory are somewhat higher than for a trajectory presented on a blank background (Westheimer \& Wehrhahn, 1994), but this is likely due to the interference of dot motions close to the trajectory (Watamaniuk et al., 1995).

The thresholds for each of the judged motions, global and trajectory, were entered into a two-factor ANOVA with the direction of the nonjudged motion as one factor and the general direction of the trajectory, up or down, as the other factor. No significant results were found in either ANOVA. When observers judged the direction of the trajectory motion, precision was unaffected by the direction of the global motion $[F(4,20)=0.58, p=.68]$ and there was no significant difference between the thresholds for the upward- and downward-moving trajectories $[F(1,20)=$ $2.46, p=.13]$. The interaction between these two factors was also insignificant $[F(4,20)=0.77, p=.55]$. When observers judged the direction of the global motion, precision was unaffected by the direction of the trajectory mo$\operatorname{tion}^{1}[F(4,20)=0.71, p=.60]$ and there was no significant difference between the thresholds obtained when the trajectories moved upward or downward $[F(1,20)=1.07, p=.31]$. The interaction between these two factors was also insignificant $[F(4,20=0.83, p=.52]$.

Since none of the effects described above reached significance, subsequent thresholds were evaluated for a single experimental run by combining performance over the direction of global flow when evaluating trajectory direction discrimination or over the direction of trajectory motion when evaluating global direction discrimination. Probit analysis was then performed on the combined performance values to determine a direction discrimination threshold.

We tested for the effects of cuing by doing independent one-way ANOVAs of the data for trajectory direction judgments and global direction judgments. Data for the three cuing conditions-precue-constant, precue-random, and postcue-random - were entered into the analysis. Each observer performed three sessions of the constant condition and five sessions of the two random conditions. Figure 3 shows the average thresholds for both trajectory and global direction discrimination for the 3 observers. Notice that for all 3 observers, the temporal position of the cue did not produce a large change in the direction discrimination thresholds. The ANOVAs confirmed this observation. The effect of cue type was insignificant for trajectory direction discrimination $[F(2,36)=0.92, p=.41]$ as well as for global direction discrimination $[F(2,36)=2.17, p=.13]$.

These results suggest that observers can simultaneously acquire and encode motion information at two different scales - one global, requiring integration over a large area, and one local, the motion of an individual dot. These results are consistent with those of Farell and Pelli (1993), who found that when asked to identify features, observers could attend to multiple scales (large and small) of features at the same time.

One possible reason why observers were able to acquire information about global and local motion is that the two kinds of motion may have been processed by mechanisms tuned to different speeds. Specifically, although all dots took the same spatial displacement for each frame, the perceived speed of the background motion and the trajectory motion differed because the background dots did not all move in the same direction. Observations from our lab show that the speed of global motion is approximately equal to the average vector in the mean direction. This means that the global motion of the background dots in the present experiment (displacement $=0.27^{\circ}$ frame, distribution of directions spanning $160^{\circ}, 60-\mathrm{Hz}$ frame rate) would appear to be moving at about $11.4 \% \mathrm{sec}$ rather than $16.2 \% \mathrm{sec}$ - the speed of the trajectory dot. This $30 \%$ difference in speed may have been sufficient for the two motion signals to be processed by different motion mechanisms and thus explain why observers can make precise judgments about either type of motion. On this point, Watamaniuk (1997) reported that detection of a dot moving on a fixed trajectory embedded in a background of global flow similar to that used here depended upon the speed of the trajectory dot. Namely, decreasing the speed of the trajectory dot to approximately the speed of global flow improved detectability substantially-from $60 \%$ to $85 \%$ for 1 observer (overall detectability was lower in Watamaniuk's study because the trajectory could appear anywhere in the display). If the "different speed" hypothesis stated above can be applied to detection as well as discrimination, the prediction would be that detection should be worse when the trajectory has the same "speed" as the global flow. Since Watamaniuk found the opposite result, when the trajectory and global flow dots have equal frame-to-frame displacements, their motions are probably processed by local detectors with similar speed tuning. Thus it is unlikely that performance in the present experiment was due to the trajectory and global flow motion 
Discrimination of Trajectory Motion

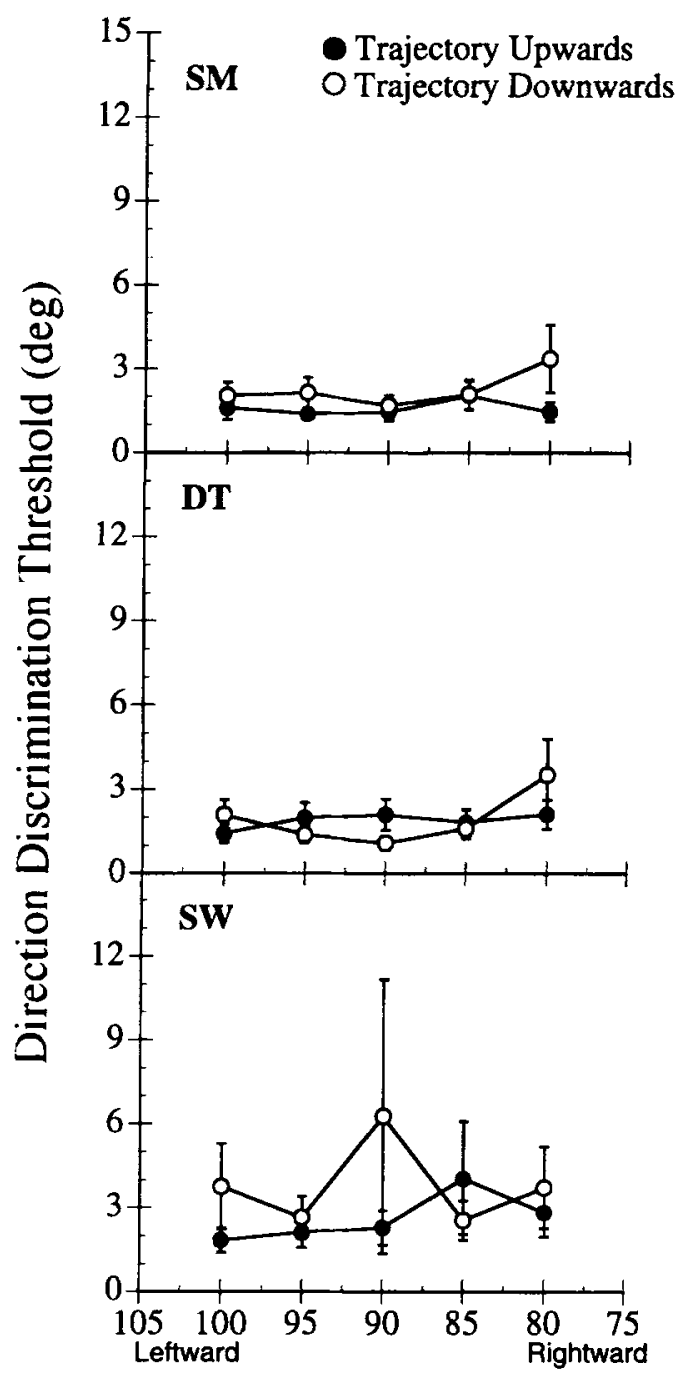

\section{Discrimination of Global Motion}

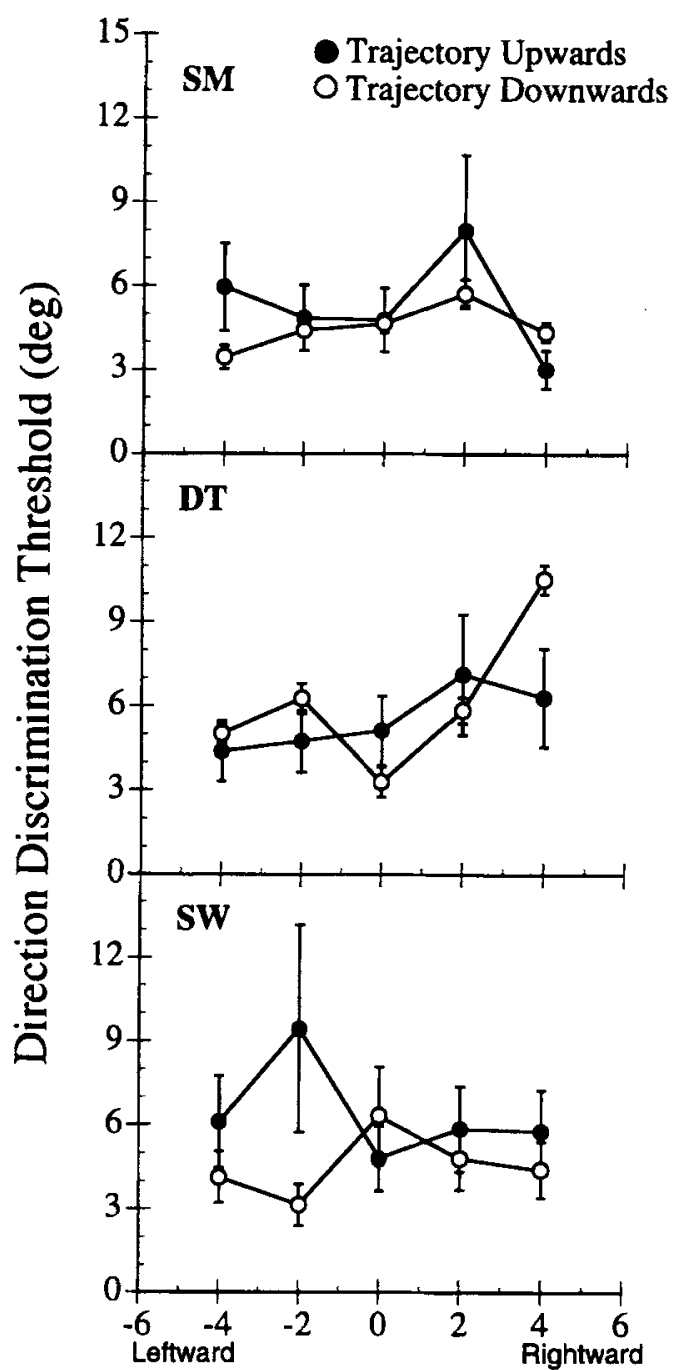

$$
\begin{gathered}
\text { Direction of } \\
\text { Trajectory Motion (deg) }
\end{gathered}
$$

Figure 2. Direction discrimination thresholds (in degrees) for the trajectory motion plotted as a function of the direction of global motion (left panel) and direction discrimination thresholds (in degrees) for the global motion plotted as a function of the direction of trajectory motion (right panel). Data are shown separately for trajectory motion upward and downward for the 3 observers. Notice that for all observers, thresholds did not depend systematically on the direction of the unjudged motion (the functions are essentially flat). In addition, discrimination thresholds were the same whether the trajectory moved upward or downward.

being processed by mechanisms tuned to different speeds. We explored two other possible explanations for our results in the next two experiments.

\section{EXPERIMENT 2 \\ Temporally Distributed Processing Hypothesis}

In this experiment, we explored the possibility that the direction discrimination performance in the precue-random and postcue-random conditions in the previous experiment was equal because the observer distributed processing time between the global flow and trajectory motion analyses. This hypothesis posits that under conditions in which the judged motion changed from trial to trial, observers used one half of the stimulus duration to process the global motion and the other half to process the trajectory motion. The idea is that even when the tone indicating which motion to judge is presented prior to the stimu- 


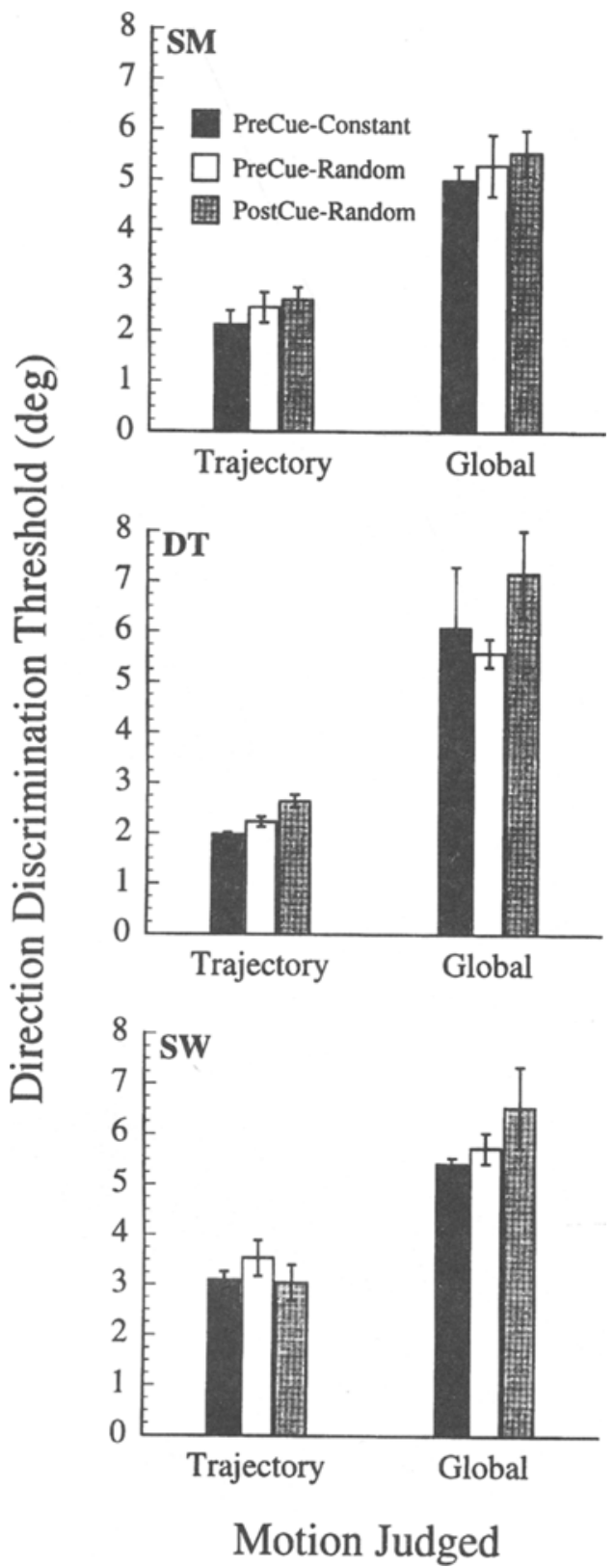

Figure 3. Trajectory and global motion direction discrimination thresholds in degrees plotted for three cuing conditions for the 3 observers. Cuing condition had no effect on discrimination performance for either type of motion.

lus, some time is required to process and interpret that tone. If this time to process the tone was longer than the $200 \mathrm{msec}$ by which it preceded the stimulus, the observer would have to process both motions so that the information for either discrimination was available when the tone was finally interpreted. This hypothesis predicts equal performance for the precue-random and postcue-random conditions, and thus it may not be surprising that in Experiment 1 these two conditions yielded similar discrimination thresholds. To test this hypothesis, we repeated the precue-constant condition but reduced the stimulus duration to one half its original value, to $85 \mathrm{msec}$ (five frames). The constant condition was used so that no trial-by-trial decisions about which motion to judge needed to be made and there was no reason for the observer to process both the local motion and the global motion to carry out the discrimination task in this condition. The distributedprocessing hypothesis would be supported if performance in the 85-msec condition was similar to that found for the random conditions with a $167-\mathrm{msec}$ duration.

\section{Method}

Observers. The same 3 observers as in the previous experiment participated in this experiment.

Stimulus and Procedure. The stimulus and procedure were identical to these used in the previous experiment with the exception that the duration of the stimulus was reduced to only $85 \mathrm{msec}$ (five frames)

\section{Results}

To determine whether the distributed-processing hypothesis could explain the results of the first experiment, an analysis compared the precue-constant 85 -msec data to the average of the precue- and postcue-random conditions. Figure 4 shows the data for the 3 observers. As can be seen in Figure 4, the $85-\mathrm{msec}$ duration resulted in an increase in the direction discrimination threshold for both the trajectory motion and the global motion for all observers, though observer D.T. seems to have been affected less by the duration change than the other 2 observers. The increase in global direction discrimination thresholds with a shorter stimulus duration is consistent with previous findings (Watamaniuk \& Sekuler, 1992). An ANOVA of these data showed that discrimination thresholds under the 85 -msec condition were significantly higher than those taken at a duration of $167 \mathrm{msec}$ for both the trajectory motion $[F(1,37)=26.8, p=.0001]$ and the global motion $[F(1,37)=17.6, p=.0002]$.

Since reducing the stimulus duration resulted in a significant increase in thresholds over the two random conditions, the distributed-processing hypothesis is not supported and cannot account for the similarity of performance across cuing conditions found in Experiment 1.

\section{EXPERIMENT 3 \\ Shrinking of the Attentional Window Hypothesis}

Since the distributed-processing hypothesis failed, we tested another possibility, namely that observers may have narrowed their attentional window so that they were not attending to the entire display. This is not a new idea. Several researchers have proposed a variable-size attentional focus or window to explain visual search performance (e.g., Nakayama, 1990). But how would narrowing one's attentional window help in the present task? One straightforward implication of narrowing one's attentional window would be to restrict the spatial extent of the incoming visual information. For example, if observers used a circular attentional window that was large enough to just en- 


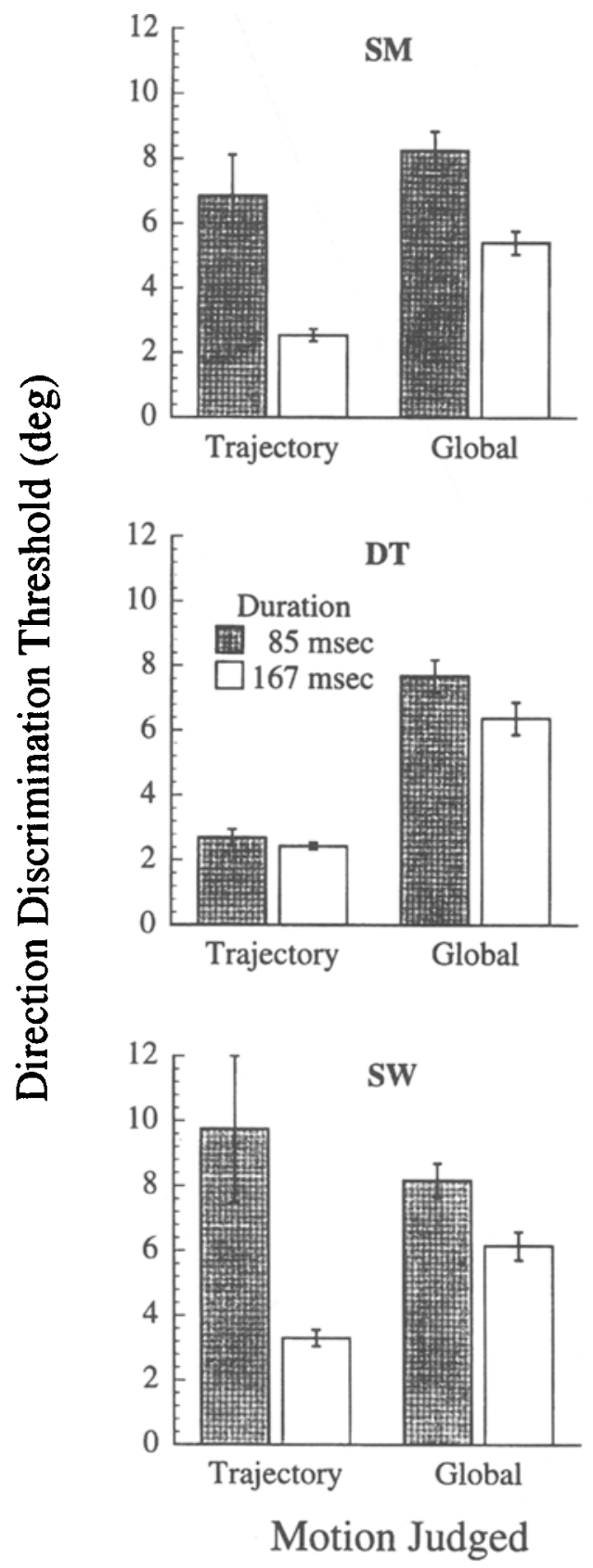

Figure 4. Trajectory and global motion direction discrimination thresholds in degrees plotted for two different stimulus durations for the 3 observers. Reducing stimulus duration to 85 msec resulted in a significant increase in discrimination thresholds for both trajectory and global motion.

compass the local trajectory (about $2.7^{\circ}$ in diameter), they would reduce the area of the display to only $7.3 \%$ of the original size $\left(78.5 \mathrm{deg}^{2}\right)$. This reduction in area could significantly decrease the amount of motion information being processed by the visual system, if unattended information is also not processed. In addition, if the trajectory is analyzed by neurons with receptive fields as large as the length of the entire trajectory, such as those in the middle temporal or medial superior temporal areas (see, e.g., Gattass \& Gross, 1981; Van Essen, Maunsell, \& Bixby, 1981), then the trajectory and the global motion may be analyzed by mechanisms with similarly sized receptive fields. This is an attractive hypothesis because it reduces the task to one of simply monitoring motion mechanisms with a certain receptive field size (a single spatial scale) for both the trajectory motion and the global motion. Of course, this hypothesis relies on the assumption that the global motion task could be performed as effectively by integrating the local motion information over an area much smaller than the entire display. Although previous research has shown that direction discrimination thresholds of global flow increase as the area of the display decreases (Watamaniuk \& Sekuler, 1992), those data were collected with longer stimulus durations. When the stimulus duration is brief, as in the present study, the global motion percept may result from the integration of only a subsample of the available motion information. Thus reducing the stimulus area may have less of a deleterious effect on global motion performance than has been observed previously.

We tested this shrinking-window-of-attention hypothesis by repeating the precue-constant condition but with a stimulus mask $3.5^{\circ}$ in diameter, which is slightly larger than the length of the trajectory $\left(2.7^{\circ}\right)$.

\section{Method}

Observers. The same 3 observers as in the previous experiment participated in this experiment.

Stimulus and Procedure. The stimulus and procedure were identical to those used in the first experiment with the exception that the stimulus was shown through an aperture mask $3.5^{\circ}$ in diameter. This allowed the observers to see only $9.6 \%$ of the dots plotted in each frame.

\section{Results}

To determine whether the attentional-window hypothesis could account for performance in the first experiment, an analysis compared the precue-constant $3.5^{\circ}$ aperture data to the average of the precue- and postcue-random conditions. Figure 5 shows the data for the 3 observers. As can be seen in Figure 5 , the $3.5^{\circ}$ aperture resulted in an increase in the direction discrimination threshold for the global motion but not for the trajectory motion for all observers. This increase in global direction discrimination thresholds with a decrease in the stimulus size is consistent with previous findings (Watamaniuk \& Sekuler, 1992). An ANOVA showed that discrimination thresholds under the $3.5^{\circ}$ aperture condition were significantly higher than those taken with a $10^{\circ}$ aperture for the global motion $[F(1,37)=53.3, p=.0001]$ but not for the trajectory motion $[F(1,37)=0.55, p=.47]$. This suggests that if observers reduced their attentional window to encompass only the trajectory in the random conditions of Experiment 1 , discrimination of the global motion would have been poorer than that obtained.

\section{GENERAL DISCUSSION}

To summarize, the present experiments show that observers are able to discriminate either the direction of a tra- 

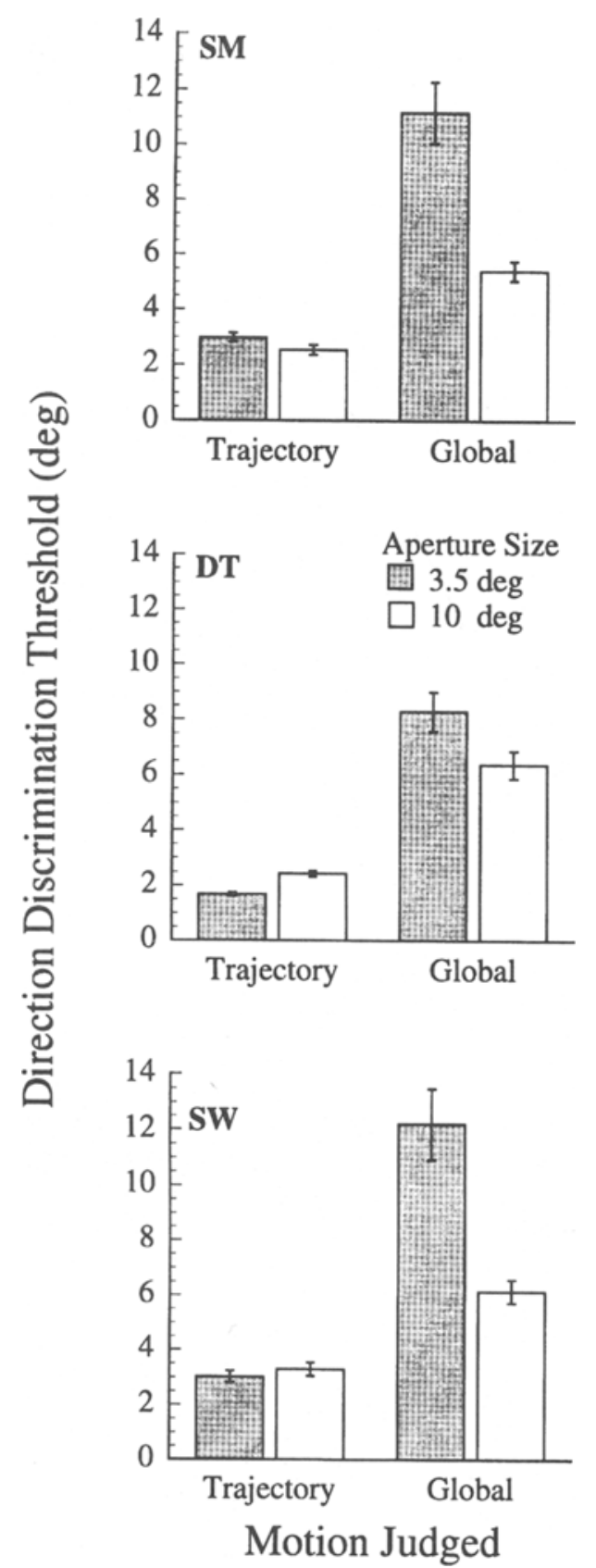

Figure 5. Trajectory and global motion direction discrimination thresholds in degrees plotted for two stimulus aperture sizes for the 3 observers. Reducing the aperture size had no effect on trajectory thresholds, but there was a significant increase in the thresholds for global motion.

jectory embedded within a global flow field or the direction of the global flow field with little interference from the direction of the unjudged motion. In addition, observers do not need to know in advance which motion they are to judge: Discrimination performance for either motion was not significantly different regardless of whether the observers were signaled before the onset of the stimulus or immediately after the offset of the stimulus. It was shown that the similarity in performance between the precue and postcue conditions was not due to distributing processing resources temporally between the two motions within a trial. Reducing the stimulus duration by one half (to $85 \mathrm{msec}$ ) and having observers always judge either the trajectory or the global motion within a trial resulted in significant increases in discrimination thresholds. The data also could not be explained by a narrowing of the attentional window. When the stimulus aperture was reduced to just a little larger than the extent of the trajectory, discrimination thresholds for global motion increased significantly. Taken together, the present experiments show that in the human visual system, direction information for both global and local motion are encoded simultaneously and that the observer has access to either motion signal after the presentation of a stimulus.

Although the present study bears some resemblance to studies on global precedence (e.g., Navon, 1977, 1981), global precedence specifically refers to a temporal advantage of the global information over the local information. That is, global features are responded to faster than local features. No explanation or theory concerning global precedence addresses the integrity of the information present at the different scales. Thus, the present study does not address the question of global precedence in a direct way. However, the present data do suggest that for motion, the visual system does not acquire information at low frequencies first and then progress to higher frequencies. We can consider the global motion of the background dots as a low spatial frequency signal because global motion depends upon the integration of motion signals over a substantial time and space (Watamaniuk et al., 1989; Williams $\&$ Sekuler, 1984). A cell responding to global motion must therefore have a relatively large receptive field that would make it selective for low rather than high spatial frequencies. We can consider the movement of a single dot as a higher spatial frequency signal through similar logic. This distinction seems to contradict the well-known fact that random dots contain energy over a broad range of spatial frequencies. However, Grzywacz, Watamaniuk, and McKee (1995) have determined the size of a motion-energy detector that would provide the best directional response for a single dot moving on a fixed trajectory embedded in a background of random-direction noise like that used in the present experiments. Their simulations showed that the best responding detector would have a receptive field diameter equal to about 4.6 times the dot's frame-to-frame displacement, which for the present experimental conditions would correspond to about $1.24^{\circ}$. It is unlikely that a cell with a receptive field that small could provide accurate information regarding the direction of global flow, especially in light of the poor performance for global direction discrimination in Experiment 3. Thus the present data suggest that motion information at low and high frequencies is coded simultaneously and without significant interference. This finding is consistent with the many psychophysical papers that support the notion that there are separate spatial frequency channels in the human visual system (e.g., Campbell \& Robson, 1968; Graham, 1985; 
Henning, Hertz, \& Broadbent, 1975; Pantle \& Sekuler, 1968; Watson \& Robson, 1981; Wilson \& Bergen, 1979). In general, these spatial channels are thought to be independent and to operate in parallel (see Olzak \& Thomas, 1986), although there can be interactions between widely differing spatial frequencies (Hirsch, Hylton, \& Graham, 1982; Olzak, 1981).

There is some controversy, however, about whether the perceived flow of a small field of dots is dependent upon low frequencies. Morgan and Fahle (1992) found that the upper displacement threshold $\left(D_{\max }\right)$ for random-dot displays was unaffected by changes in density when the elements were less than $5^{\circ}$ of arc. However, for larger element sizes, $\mathrm{D}_{\max }$ increased linearly as the density was reduced. This was taken as evidence that there was a low spatial frequency prefiltering (cutoff $\sim 3 \mathrm{c} / \mathrm{deg}$ ) of the images before motion detection. Contrasting this result, Smith, Snowden, and Milne (1994) filtered out the low frequencies in a random-dot cinematogram, using a Butterworth high-pass filter with a low spatial frequency cutoff of $12 \mathrm{c} / \mathrm{deg}$. These researchers found that this high-pass filtering did not impair direction or speed discriminations for global motion. They concluded that the global motion percept is due to an integration of local motion signals, agreeing with the original hypothesis put forth by Williams and Sekuler (1984).

Given the filtering results of Smith et al. (1994), the present task may not be one of judging motions that are input at different spatial frequencies, but rather one of retrieving motion information that has been selectively processed over different spatial extents. That is, to perceive global motion, many local motion signals must be integrated over a large area and over time. In order to identify the trajectory motion correctly, on the other hand, only the trajectory dot's motion is of interest, and the integration of any of the other dots' motions would likely lead to incorrect assessment of the trajectory's direction. Thus the present results may be interpreted as showing our ability to judge motions that have been processed over different spatial extents rather than as input at different spatial frequencies. This ability to identify motions at different scales is consistent with Farell and Pelli's (1993) finding that observers, when briefly shown a display containing rows of letters differing in size, can identify the letters in any row within a partial report paradigm.

The results of the duration experiment suggest that the observers were not attending to each motion for half of the display duration. One possible explanation for our results is that we may not need to split attention between the global and local information in order to encode bothonly the local information may need to be attended to. This situation would be useful because we are mobile creatures. As we move through the environment, stationary objects in our field of view move radially outward from our direction of heading, creating an optic flow field (Gibson, 1966). The structure of this flow field can provide the observer with information about the layout of the environment and allows the observer to navigate through it. In addition, our environment contains moving objects that move independently of our movements, and they tend to change their direction of motion slowly over time. In this regard, our stimuli of a trajectory embedded in global flow may represent a limited, laboratory version of the natural environment. Given that these two sources of motion information exist in our environment, it would be of ecological value to attend to a moving object of interest, either to capture or avoid it, while still being able to navigate competently. Of course, the preceding discussion about the effects of filtering random-dot displays is relevant here as well. If the optic flow field can be computed from the motions of local elements, this would support the notion that we do not need to attend to both high and low spatial frequency motion information, since only the highfrequency information is necessary (Smith et al., 1994). Thus the ability to detect and discriminate the direction of a trajectory embedded in global motion may reflect a difference in processing of the two motion signals because of the differences in their local motion characteristics (i.e., constant vs. changing directions). Trajectory networks that specifically respond to objects moving in constant directions have been suggested by Watamaniuk et al. (1995) and modeled by Grzywacz et al. (1995).

The final present experiment showed that observers were not limiting their attentional window to include only the area where the trajectory target could appear. When the stimulus aperture was masked down to a diameter slightly larger than the length of the trajectory, direction discrimination of the global flow was significantly reduced. This also implies that the global flow percept is dependent upon seeing a relatively large sample of the motions that define the global flow pattern from moment to moment. This is consistent with the results of Watamaniuk and Sekuler (1992), who showed that masking a randomdot display to smaller and smaller areas increased direction discrimination thresholds of global flow.

In conclusion, the present experiments show that human observers have simultaneous access to local and global motion and that no partitioning of processing resources or attention is required to guarantee highly precise information about both.

\section{REFERENCES}

Campbell, F. W., \& Robson, J. G. (1968). Application of Fourier analysis to the visibility of gratings. Journal of Physiology, 197, 551-566. Farell, B., \& PELli, D. G. (1993). Can we attend to large and small at the same time? Vision Research, 33, 2757-2772.

Finney, D. J. (1971). Probit analysis. Cambridge: Cambridge University Press.

GatTass, R., \& Gross, C. G. (1981). Visual topography of striate projection zone (MT) in posterior superior temporal sulcus of the macaque. Journal of Neurophysiology, 46, 621-638.

GrBSON, J. J. (1966). The senses considered as perceptual systems. Boston: Houghton Mifflin.

GRAHAM, N. (1985). Detection and identification of near-threshold visual patterns. Journal of the Optical Society of America A, 2, 1468-1482. Grzywacz, N.M., Watamaniuk, S.N.J., \& McKeE, S.P. (1995). Temporal coherence theory for the detection and measurement of visual motion. Vision Research, 35, 3183-3203.

Henning, G. B., Hertz, B, G., \& Broadbent, D. E. (1975). Some ex- 
periments bearing on the hypothesis that the visual system analyses spatial frequency. Vision Research, 15, 887-897.

Hirsch, J., Hylton, R., \& GRAHAM, N. (1982). Simultaneous recognition of two spatial frequency components. Vision Research, 22, 365-375.

HoFFMAN, J. E. (1980). Interaction between global and local levels of a form. Journal of Experimental Psychology: Human Perception \& Performance, 6, 222-234.

Hughes, H. C., Layton, W. M., Baird, J. C., \& Lester, L. S. (1984). Global precedence in visual pattern recognition. Perception \& Psychophysics, 35, 361-371.

LAGASSE, L. L. (1993). Effects of good form and spatial frequency on global precedence. Perception \& Psychophysics, 53, 89-105.

MARTIN, M. (1979). Local and global processing: The role of sparsity. Memory \& Cognition, 7, 476-484.

MILLER, J. (1981). Global precedence in attention and decision. Journal of Experimental Psychology: Human Perception \& Performance, 7, 1161-1174.

MORGAN, M. J., \& FAHLE, M. (1992). Effects of pattern element density upon displacement limits for motion detection in random binary luminance patterns. Proceedings of the Royal Society of London: Series $B, 248,189-198$.

NAKAYAMA, K. (1990). The iconic bottleneck and the tenuous link between early visual processing and perception. In C. Blakemore (Ed.), Vision coding and efficiency (pp. 411-422). Cambridge: Cambridge University Press.

NAvon, D. (1977). Forest before trees: The precedence of global features in visual perception. Cognitive Psychology, 9, 353-383.

Navon, D. (1981). The forest revisited: More on global precedence. Psychological Research, 43, 1-32.

NAvon, D. (1991). Testing a queue hypothesis for the processing of global and local information. Journal of Experimental Psychology: General, 120, 173-189.

Navon, D., \& Norman, J. (1983). Does global precedence really depend upon visual angle? Journal of Experimental Psychology: Human Perception \& Performance, 9, 955-965.

OLZAK, L. (1981). Inhibition and stochastic interactions in spatial pattern perception. Dissertation Abstracts International, 42, 1651B.

OlzaK, L., \& Thomas, J. P. (1986). Seeing spatial patterns. In K. R. Boff, L. Kaufman, \& J. P. Thomas (Eds.), Handbook of perception and human performance: Vol. I. Sensory processes and performance (pp. 7.1-7.56). New York: Wiley.

Pantle, A., \& Sekuler, R. (1968). Size-detecting mechanisms in human vision. Science, 162, 1146-1148.

PaQueT, L., \& MERIKLE, P. M. (1984). Global precedence: The effect of exposure duration. Canadian Journal of Psychology, 3, 45-53.

PoMERANTZ, J. R. (1983). Global and local precedence: Selective attention in form and motion perception. Journal of Experimental Psychology: General, 112, 516-540.
Robertson, L. C., EgLy, R., LAMB, M. R., \& KerTh, L. (1993). Spatial attention and cuing to global and local levels of hierarchical structure. Journal of Experimental Psychology: Human Perception \& Performance, 19, 471-487.

SMITH, A. T., SNOwden, R. J., \& Milne, A. B. (1994). Is global motion really based on spatial integration of local motion signals? Vision Research, 34, 2425-2430.

SPERLING, G. (1960). The information available in brief visual presentations. Psychological Monographs: General \& Applied, 74, 1-29.

VAn Essen, D. C., Maunsell, J. H. R., \& Bixby, J. L. (1981). The middle temporal visual area in macaque: Myeloarchitecture, connections, functional properties and topographic representation. Journal of Comparative Neurology, 199, 293-326.

WARD, L. M. (1982). Determinants of attention to local and global features of visual forms. Journal of Experimental Psychology: Human Perception \& Performance, 8, 562-581.

Ward, L. M. (1983). On processing dominance: Comment on Pomerantz. Journal of Experimental Psychology: General, 112, 541-546.

WatamaniUK, S. N. J. (1997). Speed tuning for detecting a trajectory in noise. Investigative Ophthalmology \& Visual Science, 38, S1167.

Watamaniuk, S. N. J., McKee, S. P., \& Grzywacz, N. M. (1995). Detecting a trajectory embedded in random-direction motion noise. $\mathrm{Vi}$ sion Research, 35, 65-77.

WatamaniuK, S. N. J., \& SeKULER, R. (1992). Temporal and spatial integration in dynamic random-dot stimuli. Vision Research, 32, 2341-2347.

Watamaniuk, S. N. J., Sekuler, R., \& Williams, D. G. (1989). Direction perception in complex dynamic displays: The integration of direction information. Vision Research, 29, 47-59.

WATSON, A. B., \& ROBSON, J. G. (1981). Discrimination at threshold: Labelled detectors in human vision. Vision Research, 21, 1115-1122.

WESTHEIMER, G., \& WEHRHAHN, C. (1994). Discrimination of direction of motion in human vision. Journal of Neurophysiology, 71, 33-37.

Williams, D. G., \& SeKuler, R. (1984). Coherent global motion percepts from stochastic local motions. Vision Research, 24, 55-62.

WILSON, H. R., \& BERGEN, J. R. (1979). A four mechanism model for threshold spatial vision. Vision Research, 19, 19-32.

\section{NOTE}

1. When we tested for the effect of the direction of the trajectory motion on global motion discrimination, the upward and downward trajectory directions were coded in terms of differences in direction from the cardinal direction (as plotted in Figure 2).

(Manuscript received August 28, 1996; revision accepted for publication February 12, 1997.) 